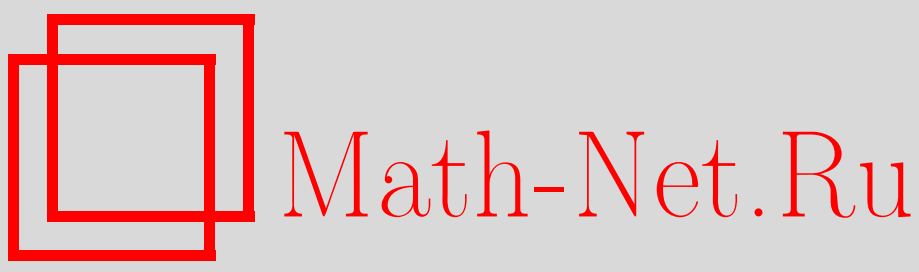

K. С. Абдухаликов, Группы автоморфизмов инвариантных решеток в модуле Стейнберга групп типа Ли нечетной характеристики, Матем. сб., 1998, том 189, номер 9, 3-22

DOI: https://doi.org/10.4213/sm345

Использование Общероссийского математического портала Math-Net.Ru подразумевает, что вы прочитали и согласны с пользовательским соглашением

http://www.mathnet.ru/rus/agreement

Параметры загрузки:

IP: 34.239 .49 .27

26 апреля 2023 г., $10: 23: 46$ 


\title{
Группы автоморфизмов инвариантных решеток в модуле Стейнберга групп типа Ли нечетной характеристики
}

\author{
Целью настоящей работы является выгисление групп автоморфизмов $G$-ин- \\ вариантных решеток в модуле Стейнберга $V$, где $G$ - простая конечная группа \\ типа Ли над полем характеристики $p>2$. \\ Библиографйл: 29 названий.
}

\section{§1. Введение}

Пусть $W$ - рациональный модуль над конечной группой $G$. Будем назьвать $\mathbb{Z}$-решетку $\Lambda \subset W$ инвариантной, если она инвариантна относительно действия группы $G$. Напомним, что число классов подобия инвариантных решеток в $W$ конечно тогда и только тогда, когда действие $G$ на $W$ абсолютно неприводимо [1]. В этом случае на пространстве $W$ сушествует единственная (с точностью до скалярного множителя) $G$-инвариантная симметрическая положительно определенная билинейная форма. Через $\operatorname{Aut}(\Lambda)$ обозначим группу изометрий $\Lambda$ относительно этой формы.

Инвариантные решетки были изучены для некоторых классов групп и их естественных модулей (см., например, [2]-[4] и особенно [5] для наиболее полного освешения предмета). В работе [6] были описаны инвариантные решетки в модуле Стейнберга групшы $G=\operatorname{PSL}_{2}(q)$ и вычислены их группы автоморфизмов. В этом случае инвариантные решетки были классифицированы с помощью квадратично-вычетных кодов над полем из двух элементов. Отметим, что по аналогии с этой работой в [3] исследованы инвариантные эрмитовы решетки в комплексном модуле Стейнберга группы $G=\operatorname{PSL}_{2}(q)$, и в этом случае для классификации решеток задействованы квадратично-вычетные коды над полем из четырех элементов.

Пусть теперь $G$ - простая конечная группа типа Ли, $V$ - модуль Стейнберга над полем $\mathbb{C}$ группы $G$. В [5] было высказано пожелание изучить инвариантные решетки в модуле $V$. Известно (см. [7]), что модуль Стейнберга неприводим. Модуль Стейнберга может быть реализован над полем рациональных чисел, но мы будем работать в модуле $V$, поскольку используем теорию комплексных характеров. Под решеткой $\Lambda \subseteq V$ будем понимать $\mathbb{Z}$-оболочку некоторого базиса. Если $\Lambda$ является $G$-инвариантной, то на пространстве $W=\Lambda \otimes_{\mathbb{Z}} \mathbb{Q}$ существует инвариантная симметрическая положительно определенная форма, относительно которой будем рассматривать $\operatorname{Aut}(\Lambda)$. Основной задачей данной работы является доказательство следующей теоремы (с использованием классификации конечных простых групп).

ТЕОРЕМА. Пусть $G$ - простая конечная группа типа Ли над полем характеристики $p>2, V$-модуль Стейнберга и $\Lambda-G$-инвариантная $\mathbb{Z}$-решетка в модуле $V$. Тогда выполняется одно из следующих утвержсений:

1) $G=\operatorname{PSL}_{2}(7), \operatorname{Aut}(\Lambda) \cong \mathbb{Z}_{2} \times \operatorname{AGL}_{3}(2), \mathbb{Z}_{2} \times \operatorname{Sp}_{6}(2)$ или $\mathbb{Z}_{2}^{7}: S_{7}$;

Работа частично поддержана грантом фонда INTAS.

(C) К. С. АвдУхаликов 1998 
2) $G=\operatorname{PSL}_{2}(23), \operatorname{Aut}(\Lambda) \cong \mathbb{Z}_{2} \times M_{24}$;

3) $G=\operatorname{PSU}_{3}(3), \operatorname{Aut}(\Lambda) \cong \mathbb{Z}_{2} \times \operatorname{Sp}_{6}(2)$;

4) $\mathbb{Z}_{2} \times G \leqslant \operatorname{Aut}(\Lambda) \leqslant \mathbb{Z}_{2} \times \operatorname{Aut}(G)$;

5) $G=\mathrm{PSL}_{2}(q), \operatorname{PSU}_{3}(q),{ }^{2} B_{2}(q),{ }^{2} G_{2}(q), G$ действует дважды транзитивно на множестве из $m$ точек, $m=\operatorname{dim} V+1$ и $\operatorname{Aut}(\Lambda) \cong \mathbb{Z}_{2} \times S_{m}$.

Мы будем использовать две леммы, представляющие самостоятельный интерес. Оказалось, что характер Стейнберга конечной простой группы типа Ли над полем характеристики $p$ является единственным неприводимым проективньм комплексным характером степени $p^{r}>1$ (за исключением некоторых групп-см. лемму 2.2). Далее, характер Стейнберга простой группы $G$ является примитивньм, за исключением групп $\mathrm{PSL}_{2}(5), \mathrm{PSL}_{2}(7), \mathrm{PSL}_{3}(2)$ и $\mathrm{PSp}_{4}(3)$.

\section{§2. Предварительные результаты}

В этом параграфе мы рассмотрим некоторые свойства модуля Стейнберга. Будем часто использовать следующий факт (см. [8], [9]):

Лемма 2.1. Пусть $q$ и $n$ - целье числа, $q \geqslant 2, n \geqslant 3,(q, n) \neq(2,6)$. Тогда

1) существует простое число $d$ такое, что $d$ делит $q^{n}-1$ и не делит $q^{i}-1$ при всех $i<n$ (d называется примитивным простым делителем числа $\left.q^{n}-1\right)$

2) если примитивный простой делитель числа $q^{n}-1$ делит $q^{m}-1$, то $n \mid m$.

Следуюшие две леммы представляют независимый интерес.

Лемма 2.2. Пусть $G$ - конечная простая группа типа Ли над полем характеристики $p, G \neq \mathrm{PSL}_{2}(4), \mathrm{PSL}_{3}(2), \mathrm{PSL}_{3}(4), \mathrm{PSL}_{4}(2), \mathrm{PSU}_{4}(2), \mathrm{PSU}_{6}(2)$, $\mathrm{Sp}_{4}(2)^{\prime}, \mathrm{Sp}_{6}(2), \Omega_{8}^{+}(2),{ }^{2} B_{2}(8),{ }^{2} E_{6}(2), F_{4}(2),{ }^{2} F_{4}(2)^{\prime}, G_{2}(4), \mathrm{PSL}_{2}(9), \mathrm{PSU}_{4}(3)$, $\Omega_{7}(3), G_{2}(3)$. Тогда характер Стейнберга группы $G$ является единственным неприводимым проективным комплексным характером степени $p^{r}>1$.

ДоКАЗАТЕЛЬСТво. Пусть $\widehat{G}$ обозначает универсальную накрывающую группу групшы $G$, т.е. $\widehat{G}^{\prime}=\widehat{G}, \widehat{G} / Z(\widehat{G}) \cong G$, и $Z(\widehat{G})$ равен мультипликатору Шура групшы $G$. Тогда по условиям леммы $\widehat{G}$ является конечной группой $\mathscr{G} F$, соответствуюшей односвязной алгебраической групше $\mathscr{G}$ и эндоморфизму Фробениуса $F$ (список исключений представляет собой список групп с нестандартньм мультипликатором Шура). Любой неприводимый проективный комплексный характер групшы $G$ поднимается до неприводимого комплексного характера групшы $\widehat{G}$. Таким образом, мы должны доказать, что любой неприводимый комплексный характер $\chi$ степени $p^{r}>1$ групшы $\widehat{G}=\mathscr{G}^{F}$ является характером Стейнберга.

Пусть $\mathscr{G}^{*}$ - дуальная группа для $\mathscr{G}$ и $G^{*}=\mathscr{G}^{*} F^{*}$ (см. [7]). $\mathscr{G}$ является связной односвязной алгебраической группой, поэтому $\mathscr{G}^{*}$ - группа присоединенного типа. Согласно классификации Люстига (см. [10]-[12]) характеру $\chi$ соответствуют полупростой элемент $s^{*} \in G^{*}$ и унипотентный характер $\psi$ группы $C_{G^{*}}\left(s^{*}\right)$. Кроме того,

$$
\operatorname{deg} \chi=\frac{|\widehat{G}|_{p^{\prime}}}{\left|C_{G^{*}}\left(s^{*}\right)\right|_{p^{\prime}}} \operatorname{deg} \psi,
$$

где $N_{p^{\prime}}$ обозначает $p^{\prime}$-часть целого числа $N$. Имеем $\left|G^{*}\right|_{p^{\prime}} /\left|C_{G^{*}}\left(s^{*}\right)\right|_{p^{\prime}}=1$, так как $\operatorname{deg} \chi=p^{r}$. Покажем, что $s^{*}=1$. Действительно, если $s^{*} \neq 1$, то 
$C_{G^{*}}\left(s^{*}\right) \neq G^{*}$. Рассмотрим унипотентный характер $\theta$ степени 1 группы $C_{G^{*}}\left(s^{*}\right)$. Тогда элемент $s^{*}$ и характер $\theta$ доставляют нетривиальньй одномерный характер групшы $\widehat{G}$, что невозможно.

Таким образом, $\operatorname{deg} \chi=\operatorname{deg} \psi$ для некоторого унипотентного характера $\psi$ группы $G^{*}$. Далее мы рассмотрим все виды группы $G$ по отдельности.

1) Предположим, что $G=\operatorname{PSL}_{n}(q)$. Тогда $\widehat{G}=\operatorname{SL}_{n}(q)$ и $G^{*}=\operatorname{PGL}_{n}(q)$. Унипотентные характеры $\mathrm{PGL}_{n}(q)$ параметризуются разбиениями $\alpha$ числа $n: \psi=\psi^{\alpha}$ (см. [7]). Пусть $\alpha=\left(\alpha_{1}, \ldots, \alpha_{m}\right), 1 \leqslant \alpha_{1} \leqslant \cdots \leqslant \alpha_{m}, \alpha_{1}+\alpha_{2}+\cdots+\alpha_{m}=n$. Положим $\lambda_{i}=\alpha_{i}+i-1$. Тогда

$$
\operatorname{deg} \psi^{\alpha}=\frac{(q-1)\left(q^{2}-1\right) \cdots\left(q^{n}-1\right) \prod_{i^{\prime}<i}\left(q^{\lambda_{i}}-q^{\lambda_{i^{\prime}}}\right)}{q^{\left(\begin{array}{c}
n-1 \\
2
\end{array}\right)+\left(\begin{array}{c}
m-2 \\
2
\end{array}\right)+\cdots} \prod_{i} \prod_{k=1}^{\lambda_{i}}\left(q^{k}-1\right)} .
$$

Если $m=1$, то $\operatorname{deg} \psi^{\alpha}=1$. Пусть $m \geqslant 2$. Случаи $(q, n)=(2,6)$ и $n=2$ рассматриваются непосредственно. Если $(q, n) \neq(2,6)$ и $n>2$, то рассмотрим примитивный простой делитель $d$ числа $q^{n}-1$. Число $d$ делит числитель дроби (1) и $\operatorname{deg} \psi^{\alpha}=p^{r}$, поэтому $d \mid \prod_{k=1}^{\lambda_{m}}\left(q^{k}-1\right)$ и $\lambda_{m} \geqslant n$. Но тогда $\lambda_{m}=\left(n-\alpha_{1}-\cdots-\alpha_{m-1}\right)+(m-1) \leqslant n$ влечет $\lambda_{m}=n$. Следовательно, $\alpha_{1}=\alpha_{2}=\cdots=\alpha_{m-1}=1, \alpha_{m}=n-m+1, \lambda_{1}=1, \lambda_{2}=2, \ldots, \lambda_{m-1}=m-1$, $\lambda_{m}=n$. Отсюда

$$
\operatorname{deg} \psi^{\alpha}=\frac{q^{\left(\begin{array}{c}
m \\
2
\end{array}\right)}\left(q^{n-m+1}-1\right)\left(q^{n-m+2}-1\right) \cdots\left(q^{n-1}-1\right)}{(q-1)\left(q^{2}-1\right) \cdots\left(q^{m-1}-1\right)} .
$$

Следовательно, $m=n, \alpha_{1}=\alpha_{2}=\cdots=\alpha_{n}=1$ и $\chi$-характер Стейнберга.

2) Предположим, что $G=\operatorname{PSU}_{n}(q)$. Тогда $\widehat{G}=\operatorname{SU}_{n}(q)$ и $G^{*}=\mathrm{PU}_{n}(q)$. Унипотентные характеры $\mathrm{PU}_{n}(q)$ параметризуются разбиениями $\alpha$ числа $n: \psi=\psi^{\alpha}$ (см. [7]). Пусть $\alpha=\left(\alpha_{1}, \ldots, \alpha_{m}\right), 1 \leqslant \alpha_{1} \leqslant \cdots \leqslant \alpha_{m}, \alpha_{1}+\alpha_{2}+\cdots+\alpha_{m}=n$. Положим $\lambda_{i}=\alpha_{i}+i-1$. Тогда

$$
\operatorname{deg} \psi^{\alpha}=\frac{(q+1)\left(q^{2}-1\right) \cdots\left(q^{n}-(-1)^{n}\right) \prod_{i^{\prime}<i}\left(q^{\lambda_{i}}-(-1)^{\lambda_{i}+\lambda_{i^{\prime}}} q^{\lambda_{i^{\prime}}}\right)}{q^{\left(\begin{array}{c}
m-1 \\
2
\end{array}\right)+\left(\begin{array}{c}
m-2 \\
2
\end{array}\right)+\cdots} \prod_{i} \prod_{k=1}^{\lambda_{i}}\left(q^{k}-(-1)^{k}\right)} .
$$

Если $m=1$, то $\operatorname{deg} \psi^{\alpha}=1$. Пусть $m \geqslant 2$.

2 a) Предположим вначале, что $n$ - нечетное. Имеем $(n, q) \neq(2,3)$, так как группа $\mathrm{PSU}_{3}(2)$ не простая. Тогда сушествует примитивный простой делитель $d$ числа $q^{2 n}-1$. Число $d$ делит числитель дроби (2) и $\operatorname{deg} \psi^{\alpha}=p^{r}$, поэтому $d \mid \prod_{k=1}^{\lambda_{m}}\left(q^{k}-(-1)^{k}\right)$ и $\lambda_{m} \geqslant n$. Но тогда $\lambda_{m}=\left(n-\alpha_{1}-\cdots-\alpha_{m-1}\right)+(m-1) \leqslant n$ влечет $\lambda_{m}=n$. Следовательно, $\alpha_{1}=\alpha_{2}=\cdots=\alpha_{m-1}=1, \alpha_{m}=n-m+1$, $\lambda_{1}=1, \lambda_{2}=2, \ldots, \lambda_{m-1}=m-1, \lambda_{m}=n$. Отсюда

$\operatorname{deg} \psi^{\alpha}=\frac{q^{\left(\begin{array}{c}m \\ 2\end{array}\right)}\left(q^{n-m+1}-(-1)^{n-m+1}\right)\left(q^{n-m+2}-(-1)^{n-m+2}\right) \cdots\left(q^{n-1}-(-1)^{n-1}\right)}{(q+1)\left(q^{2}-1\right) \cdots\left(q^{m-1}-(-1)^{m-1}\right)}$.

Следовательно, $m=n, \alpha_{1}=\alpha_{2}=\cdots=\alpha_{n}=1$ и $\chi$ - характер Стейнберга.

$2 б)$ Пусть теперь $n$ - четное, $n \geqslant 4$. Поскольку $(n, q) \neq(2,4)$, найдется примитивный простой делитель $d$ числа $q^{2(n-1)-1}$. Число $d$ делит числитель дроби (2) и $\operatorname{deg} \psi^{\alpha}=p^{r}$, поэтому $d \mid \prod_{k=1}^{\lambda_{m}}\left(q^{k}-(-1)^{k}\right)$ и $\lambda_{m} \geqslant n-1$. Но тогда $\lambda_{m}=\left(n-\alpha_{1}-\cdots-\alpha_{m-1}\right)+(m-1) \stackrel{\leqslant}{\leqslant}$ влечет $\lambda_{m}=n$ или $\lambda_{m}=n-1$. Первый случай рассматривается как в $2 \mathrm{a}$ ). Пусть $\lambda_{m}=n-1$. Тогда 


$$
\begin{gathered}
\alpha_{1}=\alpha_{2}=\cdots=\alpha_{m-2}=1, \alpha_{m-1}=2, \alpha_{m}=n-m, \lambda_{1}=1, \lambda_{2}=2, \ldots, \\
\lambda_{m-2}=m-2, \lambda_{m-1}=m, \lambda_{m}=n-1 . \text { Следовательно, } \\
\left(\operatorname{deg} \psi^{\alpha}\right)_{p^{\prime}}=\frac{\left(q^{n}-1\right)\left(q^{n-1-m}-(-1)^{n-1-m}\right) \prod_{k=1}^{m-2}\left(q^{n-1-k}-(-1)^{n-1-k}\right)}{(q+1)\left(q^{m}-(-1)^{m}\right) \prod_{k=1}^{m-2}\left(q^{k}-(-1)^{k}\right)}>1,
\end{gathered}
$$

что невозможно.

3) Предположим, что $G=\operatorname{PSp}_{2 n}(q)$ или $G=\Omega_{2 n+1}(q)$. Тогда $\widehat{G}=\operatorname{Sp}_{2 n}(q)$ или $\widehat{G}=\operatorname{Spin}_{2 n+1}(q), G^{*}=\operatorname{SO}_{2 n+1}(q)$ или $G^{*}=\operatorname{PCSp}_{2 n}(q)$ соответственно. Унипотентные характеры группы $G^{*}$ параметризуются символами вида

$$
\left(\begin{array}{c}
\lambda \\
\mu
\end{array}\right)=\left(\begin{array}{llllll}
\lambda_{1} & & \lambda_{2} & \ldots & & \lambda_{a} \\
& \mu_{1} & & \ldots & \mu_{b}
\end{array}\right)
$$

где $0 \leqslant \lambda_{1}<\lambda_{2}<\cdots<\lambda_{a}, 0 \leqslant \mu_{1}<\mu_{2}<\cdots<\mu_{b}, a-b$ - нечетное и положительное, $\left(\lambda_{1}, \mu_{1}\right) \neq(0,0)$ и

$$
\sum_{i} \lambda_{i}+\sum_{j} \mu_{j}-\left[\left(\frac{a+b-1}{2}\right)^{2}\right]=n .
$$

Целое число $n$ называется рангом символа $\left(\begin{array}{c}\lambda \\ \mu\end{array}\right)$. Для унипотентного характера $\psi^{\lambda, \mu}$ имеем

$$
\operatorname{deg} \psi^{\lambda, \mu}=\frac{\prod_{k=1}^{n}\left(q^{2 k}-1\right) \prod_{i^{\prime}<i}\left(q^{\lambda_{i}}-q^{\lambda_{i^{\prime}}}\right) \prod_{j^{\prime}<j}\left(q^{\mu_{j}}-q^{\mu_{j^{\prime}}}\right) \prod_{i, j}\left(q^{\lambda_{i}}+q^{\mu_{j}}\right)}{2^{\left[\frac{a+b-1}{2}\right]} q^{\left({ }^{a+b-2}\right)+\left({ }_{2}^{a+b-4}\right)+\cdots} \prod_{i} \prod_{k=1}^{\lambda_{i}}\left(q^{2 k}-1\right) \prod_{j} \prod_{k=1}^{\mu_{j}}\left(q^{2 k}-1\right)}
$$

Так как $(q, n) \neq(2,3)$ и $n>2$, то рассмотрим примитивный простой делитель $d$ числа $q^{2 n-1}$. Число $d$ делит числитель дроби (3) и $\operatorname{deg} \psi^{\lambda, \mu}=p^{r}$, поэтому $2 \lambda_{a} \geqslant 2 n$ или $\mu_{b} \geqslant 2 n$. Рассмотрим возможные случаи.

3а) Пусть $\lambda_{a} \geqslant n, \lambda_{1} \neq 0$. Тогда

$$
\begin{aligned}
\sum & \lambda_{i}+\sum \mu_{j}-\left(\frac{a+b-1}{2}\right)^{2} \\
& \geqslant\left(1+2+\cdots+a-1+\lambda_{a}\right)+(0+1+\cdots+b-1)-\frac{(a+b-1)^{2}}{4} \\
& =\frac{(a-b)^{2}-1}{4}+\lambda_{a} \geqslant \lambda_{a} .
\end{aligned}
$$

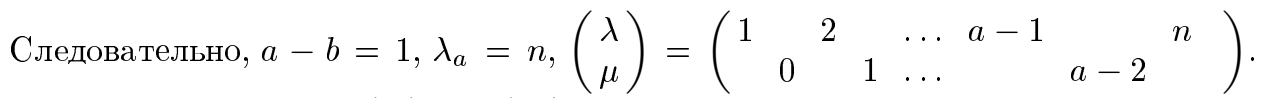

Если $b=0$, то $a=1,\left(\begin{array}{c}\lambda \\ \mu\end{array}\right)=\left(\begin{array}{c}n \\ -\end{array}\right), \operatorname{deg} \psi^{\lambda, \mu}=1$. Если $b \neq 0$, то $d \mid q^{\lambda_{a}}+q^{\mu_{1}}$ $=q^{n}+1,2 \lambda_{a-1} \geqslant 2 n$ или $2 \mu_{b} \geqslant 2 n$, что невозможно.

3б) Пусть $\lambda_{a} \geqslant n, \mu_{1} \neq 0$. Тогда

$$
\begin{aligned}
\sum & \lambda_{i}+\sum \mu_{j}-\left(\frac{a+b-1}{2}\right)^{2} \\
\geqslant & \left(0+1+\cdots+a-2+\lambda_{a}\right)+(1+2+\cdots+b)-\frac{(a+b-1)^{2}}{4} \\
& =\frac{(a-b)(a-b-4)+3}{4}+\lambda_{a} \geqslant \lambda_{a} .
\end{aligned}
$$


Следовательно, $a-b=1$ или $a-b=3, \lambda_{a}=n$. Если $a-b=1$, то $\left(\begin{array}{l}\lambda \\ \mu\end{array}\right)=$ $\left(\begin{array}{lllllll}0 & & 1 & & \ldots & b-1 & \\ & 1 & 2 & \ldots & & b\end{array}\right)$. Мы можем предполагать, что $n>2$ и $(q, n) \neq(2,4)$ (случай $n=2$ проверяется непосредственно, для $(q, n)=(2,4)$ см. [13]). Пусть $t-$ примитивный простой делитель числа $q^{2(n-1)}-1$. Тогда $t \mid q^{\lambda_{a}}+q^{\mu_{1}}=q^{n}+q$, $2 \lambda_{a-1} \geqslant 2(n-1)$ или $2 \mu_{b} \geqslant 2(n-1)$. Отсюда $b \geqslant n-1$. При $b=n-1$ имеем $t \mid q^{\lambda_{a}}+q^{\mu_{1}}$ и $t \mid q^{\lambda_{1}}+q^{\mu_{b}}$, что невозможно. При $b=n$ получаем характер Стейнберга.

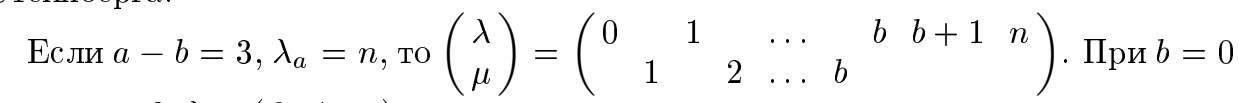
имеем $a=3, \lambda=\left(\begin{array}{lll}0 & 1 & n\end{array}\right)$,

$$
\operatorname{deg} \psi^{\lambda, \mu}=\frac{q\left(q^{n}-1\right)\left(q^{n-1}-1\right)}{2(q+1)}
$$

Поэтому $b>0, n>2$. Пусть $t$-примитивный простой делитель числа $q^{2(n-1)}-1$. Тогда $t \mid q^{\lambda_{a}}+q^{\mu_{1}}=q^{n}+q, 2 \lambda_{a-1} \geqslant 2(n-1)$ или $2 \mu_{b} \geqslant 2(n-1)$. Отсюда $b=n-2$, $a=n+1$ и

$$
\operatorname{deg} \psi^{\lambda, \mu}=\frac{q^{(n-1)^{2}}\left(q^{n}-1\right)\left(q^{n-1}-1\right)}{2(q+1)} .
$$

3в) Пусть $\mu_{b} \geqslant n, \lambda_{1} \neq 0$. Тогда

$$
\begin{aligned}
\sum & \lambda_{i}+\sum \mu_{j}-\left(\frac{a+b-1}{2}\right)^{2} \\
& \geqslant(1+2+\cdots+a)+\left(0+1+\cdots+b-2+\mu_{b}\right)-\frac{(a+b-1)^{2}}{4} \\
& =\frac{(a-b)^{2}+3}{4}+(a-b)+\mu_{b}>n .
\end{aligned}
$$

Зг) Пусть $\mu_{b} \geqslant n, \mu_{1} \neq 0$. Тогда

$$
\begin{aligned}
\sum & \lambda_{i}+\sum \mu_{j}-\left(\frac{a+b-1}{2}\right)^{2} \\
& \geqslant(0+1+\cdots+a-1)+\left(1+2+\cdots+b-1+\mu_{b}\right)-\frac{(a+b-1)^{2}}{4} \\
& =\frac{(a-b)^{2}-1}{4}+\mu_{b} \geqslant \mu_{b} .
\end{aligned}
$$

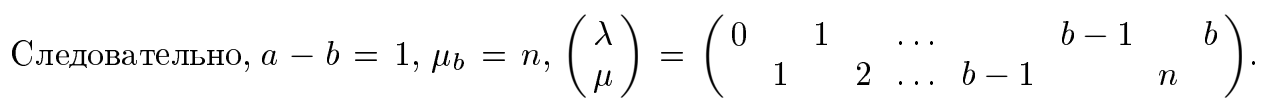
Так как $d \mid q^{\lambda_{1}}+q^{\mu_{b}}$, то $2 \lambda_{a} \geqslant 2 n$ или $2 \mu_{b-1} \geqslant 2 n$. Поэтому $b=n$ и $\chi-$ характер Стейнберга.

4) Предположим, что $G=\mathrm{P} \Omega_{2 n}^{-}(q), n \geqslant 4$. Тогда $\widehat{G}=\operatorname{Spin}_{2 n}^{-}(q)$ и $G^{*}=$ $\mathrm{P}\left(\mathrm{CO}_{2 n}^{-}(q)^{0}\right)$. Унипотентные характеры группы $G^{*}$ параметризуются символами вида

$$
\left(\begin{array}{l}
\lambda \\
\mu
\end{array}\right)=\left(\begin{array}{llll}
\lambda_{1} & \lambda_{2} & \ldots & \lambda_{a} \\
\mu_{1} & \mu_{2} & \ldots & \mu_{b}
\end{array}\right)
$$


где $0 \leqslant \lambda_{1}<\lambda_{2}<\cdots<\lambda_{a}, 0 \leqslant \mu_{1}<\mu_{2}<\cdots<\mu_{b},\left(\lambda_{1}, \mu_{1}\right) \neq(0,0), a-b \equiv 2$ $(\bmod 4), a>b$ и

$$
\sum_{i} \lambda+\sum_{j} \mu-\left[\left(\frac{a+b-1}{2}\right)^{2}\right]=n .
$$

Тогда степень унипотентного характера $\psi^{\lambda, \mu}$ равна

$$
\frac{\left(q^{n}+1\right) \prod_{k=1}^{n-1}\left(q^{2 k}-1\right) \prod_{i^{\prime}<i}\left(q^{\lambda_{i}}-q^{\lambda_{i^{\prime}}}\right) \prod_{j^{\prime}<j}\left(q^{\mu_{j}}-q^{\mu_{j^{\prime}}}\right) \prod_{i, j}\left(q^{\lambda_{i}}+q^{\mu_{j}}\right)}{2^{\frac{a+b-2}{2}} q^{\left({ }^{a+b-2}\right)+\left({ }^{a+b-4}\right)+\cdots} \prod_{i} \prod_{k=1}^{\lambda_{i}}\left(q^{2 k}-1\right) \prod_{j} \prod_{k=1}^{\mu_{j}}\left(q^{2 k}-1\right)} .
$$

Заметим, что $\left[\left(\frac{a+b-1}{2}\right)^{2}\right]=\left(\frac{a+b-1}{2}\right)^{2}-\frac{1}{4}$. Пусть $d$ - примитивный простой делитель числа $q^{2 n}-1$. Число $d$ делит числитель дроби (4) и $\operatorname{deg} \psi^{\lambda, \mu}=p^{r}$, поэтому $2 \lambda_{a} \geqslant 2 n$ или $2 \mu_{b} \geqslant 2 n$. Рассмотрим возможные случаи.

4а) Пусть $\lambda_{a} \geqslant n, \lambda_{1} \neq 0$. Тогда

$$
\begin{aligned}
\sum & \lambda_{i}+\sum \mu_{j}-\left[\left(\frac{a+b-1}{2}\right)^{2}\right] \\
& \geqslant\left(1+2+\cdots+a-1+\lambda_{a}\right)+(0+1+\cdots+b-1)-\frac{(a+b-1)^{2}}{4}+\frac{1}{4} \\
& =\frac{(a-b)^{2}}{4}+\lambda_{a}>\lambda_{a},
\end{aligned}
$$

что невозможно.

4б) Пусть $\lambda_{a} \geqslant n, \mu_{1} \neq 0$. Тогда

$$
\begin{aligned}
\sum & \lambda_{i}+\sum \mu_{j}-\left[\left(\frac{a+b-1}{2}\right)^{2}\right] \\
\geqslant & \left(0+1+\cdots+a-2+\lambda_{a}\right)+(1+2+\cdots+b)-\frac{(a+b-1)^{2}}{4}+\frac{1}{4} \\
& =\frac{(a-b)(a-b-4)+4}{4}+\lambda_{a} \geqslant \lambda_{a} \geqslant n .
\end{aligned}
$$

Следовательно, $a-b=2, \lambda_{a}=n$. Тогда $\left(\begin{array}{c}\lambda \\ \mu\end{array}\right)=\left(\begin{array}{ccccccc}0 & 1 & 2 & \ldots & b-1 & b & n \\ 1 & 2 & 3 & \ldots & b & \end{array}\right)$. Если $b=0$, то $\left(\begin{array}{l}\lambda \\ \mu\end{array}\right)=\left(\begin{array}{cc}0 & n \\ -\end{array}\right)$ и $\operatorname{deg} \psi^{\lambda, \mu}=1$. Пусть $b>0$. Тогда $a=b+2$, $a+b-2=2 b$.

Мы можем предполагать, что $(q, n) \neq(2,4)($ для $(q, n)=(2,4)$ см. [13]). Пусть $t$ - примитивньй простой делитель числа $q^{2(n-1)}-1$. Тогда $t \mid q^{\lambda_{a}}+q^{\mu_{1}}, 2 \lambda_{a-1} \geqslant$ $2(n-1)$ или $2 \mu_{b} \geqslant 2(n-1)$. Отсюда $b=n-1$ и $\chi$ - характер Стейнберга.

4 в) Пусть $\mu_{b} \geqslant n, \lambda_{1} \neq 0$. Тогда

$$
\begin{aligned}
\sum & \lambda_{i}+\sum \mu_{j}-\left[\left(\frac{a+b-1}{2}\right)^{2}\right] \\
& \geqslant(1+2+\cdots+a)+\left(0+1+\cdots+b-2+\mu_{b}\right)-\frac{(a+b-1)^{2}}{4}+\frac{1}{4} \\
& =\frac{(a-b)(a-b+4)}{4}+1+\mu_{b}>n .
\end{aligned}
$$


4 г) Пусть $\mu_{b} \geqslant n, \mu_{1} \neq 0$. Тогда

$$
\begin{aligned}
\sum & \lambda_{i}+\sum \mu_{j}-\left[\left(\frac{a+b-1}{2}\right)^{2}\right] \\
& \geqslant(0+1+\cdots+a-1)+\left(1+2+\cdots+b-1+\mu_{b}\right)-\frac{(a+b-1)^{2}}{4}+\frac{1}{4} \\
& =\frac{(a-b)^{2}}{4}+\mu_{b}>\mu_{b} \geqslant n .
\end{aligned}
$$

5) Предположим, что $G=\mathrm{P} \Omega_{2 n}^{+}(q), n \geqslant 4$. Тогда $\widehat{G}=\operatorname{Spin}_{2 n}(q)$ и $G^{*}=$ $\mathrm{P}\left(\mathrm{CO}_{2 n}^{+}(q)^{0}\right)$. Унипотентные характеры группы $G^{*}$ параметризуются символами вида

$$
\left(\begin{array}{c}
\lambda \\
\mu
\end{array}\right)=\left(\begin{array}{llll}
\lambda_{1} & \lambda_{2} & \ldots & \lambda_{a} \\
\mu_{1} & \mu_{2} & \ldots & \mu_{b}
\end{array}\right)
$$

где $0 \leqslant \lambda_{1}<\lambda_{2}<\cdots<\lambda_{a}, 0 \leqslant \mu_{1}<\mu_{2}<\cdots<\mu_{b},\left(\lambda_{1}, \mu_{1}\right) \neq(0,0), a-b \equiv 0$ $(\bmod 4)$ и

$$
\sum_{i} \lambda+\sum_{j} \mu-\left[\left(\frac{a+b-1}{2}\right)^{2}\right]=n .
$$

Тогда степень унипотентного характера $\psi^{\lambda, \mu}$ равна

$$
\frac{\left(q^{n}-1\right) \prod_{k=1}^{n-1}\left(q^{2 k}-1\right) \prod_{i^{\prime}<i}\left(q^{\lambda_{i}}-q^{\lambda_{i^{\prime}}}\right) \prod_{j^{\prime}<j}\left(q^{\mu_{j}}-q^{\mu_{j^{\prime}}}\right) \prod_{i, j}\left(q^{\lambda_{i}}+q^{\mu_{j}}\right)}{2^{c} q^{\left({ }^{a+b-2}\right)+\left({ }_{2}^{a+b-4}\right)+\cdots} \prod_{i} \prod_{k=1}^{\lambda_{i}}\left(q^{2 k}-1\right) \prod_{j} \prod_{k=1}^{\mu_{j}}\left(q^{2 k}-1\right)},
$$

где $c=(a+b-2) / 2$ при $\lambda \neq \mu$ и $c=a$ при $\lambda=\mu$. Кроме того, если $\lambda=\mu$, то символ $\left(\begin{array}{c}\lambda \\ \mu\end{array}\right)$ определяет два характера, а если $\lambda \neq \mu$, то символы $\left(\begin{array}{c}\lambda \\ \mu\end{array}\right)$ и $\left(\begin{array}{c}\mu \\ \lambda\end{array}\right)$ определяют один характер. Будем считать, что $a \geqslant b$.

Заметим, что $\left[\left(\frac{a+b-1}{2}\right)^{2}\right]=\left(\frac{a+b-1}{2}\right)^{2}-\frac{1}{4}$. Так как $(q, n) \neq(2,4)$, то найдется примитивный простой делитель $d$ числа $q^{2(n-1)}-1$. Число $d$ делит числитель дроби (5) и $\operatorname{deg} \psi^{\lambda, \mu}=p^{r}$, поэтому $2 \lambda_{a} \geqslant 2(n-1)$ или $2 \mu_{b} \geqslant 2(n-1)$. Рассмотрим возможные случаи.

5а) Пусть $\lambda_{a} \geqslant n-1, \lambda_{1} \neq 0$. Тогда

$$
\begin{aligned}
\sum & \lambda_{i}+\sum \mu_{j}-\left[\left(\frac{a+b-1}{2}\right)^{2}\right] \\
& \geqslant\left(1+2+\cdots+a-1+\lambda_{a}\right)+(0+1+\cdots+b-1)-\frac{(a+b-1)^{2}}{4}+\frac{1}{4} \\
& =\frac{(a-b)^{2}}{4}+\lambda_{a} \geqslant \lambda_{a} .
\end{aligned}
$$

Следовательно, $a=b$ и $\lambda_{a}=n-1, n$. Возможны 3 случая.

i) $\left(\begin{array}{l}\lambda \\ \mu\end{array}\right)=\left(\begin{array}{cccccc}1 & 2 & 3 & \ldots & b-1 & n \\ 0 & 1 & 2 & \ldots & b-2 & b-1\end{array}\right)$. При $b=1$ имеем $\operatorname{deg} \psi^{\lambda, \mu}=1$. Пусть $b \geqslant 2$. Так как $d \mid q^{\lambda_{a}}+q^{\mu_{2}}$, то $2 \lambda_{a-1} \geqslant 2(n-1)$ или $2 \mu_{b} \geqslant 2(n-1)$. Отсюда получаем, что $b=n$ и $\chi$ - характер Стейнберга. 
ii) $\left(\begin{array}{c}\lambda \\ \mu\end{array}\right)=\left(\begin{array}{cccccc}1 & 2 & \ldots & b-2 & b & n-1 \\ 0 & 1 & \ldots & b-3 & b-2 & b-1\end{array}\right)$. Так как $d \mid q^{\lambda_{a}}+q^{\mu_{1}}$, то $2 \lambda_{a-1} \geqslant$ $2(n-1)$ или $2 \mu_{b} \geqslant 2(n-1)$, что невозможно.

iii) $\left(\begin{array}{l}\lambda \\ \mu\end{array}\right)=\left(\begin{array}{lllllc}1 & 2 & \ldots & b-2 & b-1 & n-1 \\ 0 & 1 & \ldots & b-3 & b-2 & b\end{array}\right)$. Так как $d \mid q^{\lambda_{a}}+q^{\mu_{1}}$, то $2 \lambda_{a-1} \geqslant$ $2(n-1)$ или $2 \mu_{b} \geqslant 2(n-1)$. Отсюда получаем, что $b=n-1$ и

$$
\operatorname{deg} \psi^{\lambda, \mu}=\frac{q^{n^{2}-3 n+3}\left(q^{n}-1\right)\left(q^{n-2}+1\right)}{(q-1)(q+1)} .
$$

5б) Пусть $\lambda_{a} \geqslant n-1, \mu_{1} \neq 0$. Тогда

$$
\begin{aligned}
\sum & \lambda_{i}+\sum \mu_{j}-\left[\left(\frac{a+b-1}{2}\right)^{2}\right] \\
\geqslant & \left(0+1+\cdots+a-2+\lambda_{a}\right)+(1+2+\cdots+b)-\frac{(a+b-1)^{2}}{4}+\frac{1}{4} \\
& =\frac{(a-b)(a-b-4)+4}{4}+\lambda_{a} \geqslant n .
\end{aligned}
$$

Следовательно, $\lambda_{a}=n-1, a=b$ или $a=b+4$,

$$
\operatorname{deg} \psi^{\alpha, \mu}=\frac{\left(q^{n}-1\right) \prod_{i^{\prime}<i}\left(q^{\lambda_{i}}-q^{\lambda_{i^{\prime}}}\right) \prod_{j^{\prime}<j}\left(q^{\mu_{j}}-q^{\mu_{j^{\prime}}}\right) \prod_{i, j}\left(q^{\lambda_{i}}+q^{\mu_{j}}\right)}{2^{c} q^{\left({ }_{2}^{a+b-2}\right)+\left({ }_{2}^{a+b-4}\right)+\cdots} \prod_{i=1}^{a-1} \prod_{k=1}^{\lambda_{i}}\left(q^{2 k}-1\right) \prod_{j} \prod_{k=1}^{\mu_{j}}\left(q^{2 k}-1\right)}
$$

Пусть $a=b$. Тогда $\left(\begin{array}{c}\lambda \\ \mu\end{array}\right)=\left(\begin{array}{lllllc}0 & 1 & 2 & \ldots & b-2 & n-1 \\ 1 & 2 & 3 & \ldots & b-1 & b\end{array}\right)$. Случай $(q, n)=(2,5)$ рассматривается непосредственно, поэтому предположим, что $(q, n) \neq(2,5)$. Тогда найдется примитивный простой делитель $t$ числа $q^{2(n-2)}-1$, поэтому из (6) имеем $2 \lambda_{a-1} \geqslant 2(n-2)$ или $2 \mu_{b} \geqslant 2(n-2)$. Отсюда $b=n-2, n-1, n$. Если $b=n$, то получаем характер Стейнберга. Если $b=n-2$, то $t \mid q^{\lambda_{a}}+q^{\mu_{1}}$ и $t \mid q^{\lambda_{1}}+q^{\mu_{b}}$, но знаменатель дроби (6) не делится на $t^{2}$. Если $b=n-1$, то $\left(\begin{array}{l}\lambda \\ \mu\end{array}\right)=\left(\begin{array}{cccccc}0 & 1 & 2 & \ldots & n-3 & n-1 \\ 1 & 2 & 3 & \ldots & n-2 & n-1\end{array}\right)$. Тогда $\left(\begin{array}{c}\lambda^{\prime} \\ \mu^{\prime}\end{array}\right)=\left(\begin{array}{lllll}0 & 1 & \ldots & n-3 & n-2 \\ 1 & 2 & \ldots & n-2 & n-1\end{array}\right)-$ символ ранга $n-1$ и

$$
\operatorname{deg} \psi^{\lambda, \mu}=\frac{\left(q^{n}-1\right)\left(q^{n-2}+1\right)}{2(q-1)} \operatorname{deg} \psi^{\lambda^{\prime}, \mu^{\prime}}
$$

не может быть степенью $p$.

Пусть $a=b+4, \lambda_{a}=n-1$. Тогда

$$
\left(\begin{array}{l}
\lambda \\
\mu
\end{array}\right)=\left(\begin{array}{cccccccc}
0 & 1 & \ldots & b-1 & b & b+1 & b+2 & n-1 \\
1 & 2 & \ldots & b & & & &
\end{array}\right) .
$$

Если $b=0$, то $a=4$ и

$$
\operatorname{deg} \psi^{\lambda, \mu}=\frac{q^{3}\left(q^{n}-1\right)\left(q^{n-1}-1\right)\left(q^{n-2}-1\right)\left(q^{n-3}-1\right)}{2(q+1)^{2}\left(q^{2}+1\right)}
$$

Случай $(q, n)=(2,5)$ рассматривается непосредственно, поэтому предположим, что $(q, n) \neq(2,5)$. Тогда найдется примитивный простой делитель $t$ числа 
$q^{2(n-2)}-1$. Число $t$ делит $q^{\lambda_{a}}+q^{\mu_{1}}$, поэтому из (6) имеем $2 \lambda_{a-1} \geqslant 2(n-2)$ или $2 \mu_{b} \geqslant 2(n-2)$. Отсюда $b=n-4$ и

$$
\operatorname{deg} \psi^{\lambda, \mu}=\frac{q^{n^{2}-5 n+7}\left(q^{n}-1\right)\left(q^{n-1}-1\right)\left(q^{n-2}-1\right)\left(q^{n-3}-1\right)}{2(q+1)^{2}\left(q^{2}+1\right)} .
$$

5в) Пусть $\mu_{b} \geqslant n-1, \lambda_{1} \neq 0$. Тогда

$$
\begin{aligned}
\sum & \lambda_{i}+\sum \mu_{j}-\left[\left(\frac{a+b-1}{2}\right)^{2}\right] \\
& \geqslant(1+2+\cdots+a)+\left(0+1+\cdots+b-2+\mu_{b}\right)-\frac{(a+b-1)^{2}}{4}+\frac{1}{4} \\
& =\frac{(a-b)(a-b+4)}{4}+1+\mu_{b} \geqslant n .
\end{aligned}
$$

Следовательно, $a=b$. Заменяя символ $\left(\begin{array}{c}\lambda \\ \mu\end{array}\right)$ символом $\left(\begin{array}{c}\mu \\ \lambda\end{array}\right)$, получаем случай $5 б)$.

5г) Пусть $\mu_{b} \geqslant n-1, \mu_{1} \neq 0$. Тогда

$$
\begin{aligned}
\sum & \lambda_{i}+\sum \mu_{j}-\left[\left(\frac{a+b-1}{2}\right)^{2}\right] \\
& \geqslant(0+1+\cdots+a-1)+\left(1+2+\cdots+b-1+\mu_{b}\right)-\frac{(a+b-1)^{2}}{4}+\frac{1}{4} \\
& =\frac{(a-b)^{2}}{4}+\mu_{b} \geqslant \mu_{b} .
\end{aligned}
$$

Следовательно, $a=b$. Заменяя символ $\left(\begin{array}{c}\lambda \\ \mu\end{array}\right)$ символом $\left(\begin{array}{c}\mu \\ \lambda\end{array}\right)$, получаем случай $5 \mathrm{a})$.

6) Предположим, что $G$ - исключительная группа. Тогда утверждение леммы следует из проверки списка степеней унипотентных характеров (см. [7]). Лемма доказана.

Лемма 2.3. Пусть $G$ - конечная простая группа типа Ли, $G \neq \mathrm{Sp}_{4}(2)^{\prime}$, ${ }^{2} F_{4}(2)^{\prime}$. Характер Стейнберга группы $G$ является импримитивным характером тогда и только тогда, когда $G=\mathrm{PSL}_{2}(5), \mathrm{PSL}_{2}(7), \mathrm{PSL}_{3}(2)$ или $\mathrm{PSp}_{4}(3)$.

ДокАЗАТЕЛЬСтво. Предположим, что характер Стейнберга $\chi$ является импримитивным характером: $\chi=\operatorname{ind}_{K}^{G} \psi=\psi^{G}$, где $\psi$ - неприводимый характер группы $K$ и $[G: K]=p^{r}$. Согласно [14] возможны следуюшие случаи.

1) $G=\mathrm{PSL}_{4}(2), K \cong A_{7},[G: K]=8$, но группа $A_{7}$ не имеет неприводимых характеров степени 8 .

2) $G=\mathrm{PSL}_{2}(11), K \cong A_{5},[G: K]=11$, но единственным одномерным характером $\psi$ группы $A_{5}$ является тривиальньй характер, поэтому $\psi^{G}$ приводим.

3) $G=\mathrm{PSL}_{3}(2), K \cong 7: 3,[G: K]=8$. Пусть $\psi$ - нетривиальный одномерный характер группы $K$. Тогда по теореме Фробениуса о взаимности и согласно [13] имеем:

$$
\left[\psi^{G}, \chi\right]_{G}=\left[\psi,\left.\chi\right|_{K}\right]_{K}=\frac{1}{|K|} \sum_{x \in K} \psi(x) \bar{\chi}(x)=1 .
$$

Следовательно, $\chi=\psi^{G}$. 
4) $G=\mathrm{PSL}_{2}(5), K \cong A_{4} \cong V_{4}: 3,[G: K]=5$. Пусть $\psi$ - нетривиальный одномерный характер группы $K$. Тогда по теореме Фробениуса и согласно [13] имеем: $\left[\psi^{G}, \chi\right]_{G}=\left[\psi,\left.\chi\right|_{K}\right]_{K}=1$, поэтому $\chi=\psi^{G}$.

5) $G=\mathrm{PSL}_{2}(7), K \cong S_{4},[G: K]=7$. Пусть $\psi(x)=\operatorname{sgn}(x)-$ нетривиальный одномерный характер групшы $K$. Тогда $\left[\psi^{G}, \chi\right]_{G}=\left[\psi,\left.\chi\right|_{K}\right]_{K}=1$ и $\chi=\psi^{G}$.

6) $G=\operatorname{PSp}_{4}(3), K \cong 2^{4}: A_{5},[G: K]=27$. Пусть $\psi$ - трехмерный характер групшы $K$. Тогда $\left[\psi^{G}, \chi\right]_{G}=\left[\psi,\left.\chi\right|_{K}\right]_{K}=1$ и $\chi=\psi^{G}$.

\section{§3. Группы автоморфизмов инвариантных решеток}

В этом параграфе мы будем придерживаться следующих обозначений. $G$ является конечной простой группой типа Ли над полем $\mathbb{F}_{q}, q=p^{f}, p>2, \Lambda$ является $G$-инвариантной $\mathbb{Z}$-решеткой в модуле Стейнберга $V, H=\operatorname{Aut}(\Lambda), \chi$ - характер групшы $H$, порожденный модулем $V$. Далее, $\operatorname{sol}(L), \operatorname{abel}(L), \operatorname{soc}(L)$ обозначают максимальную разрешимую нормальную подгруппу, максимальную абелеву нормальную подгруппу и произведение всех минимальных нормальных подгрупп группы $L$ соответственно. Если $M$ - подгруппа групшы $L$ и $\psi$ - характер $L$, то $\left.\psi\right|_{M}$ обозначает ограничение $\psi$ на подгруппу $M$. Ясно, что отображение $\eta(x)=-x$ является автоморфизмом решетки $\Lambda$. Обозначим $Z=\langle\eta\rangle \cong \mathbb{Z}_{2}$.

Лемма 3.1. Если $G \neq \mathrm{PSL}_{2}(7)$, mo $Z(H)=\operatorname{abel}(H)=C_{H}(G)=Z$.

ДокАЗАТЕЛЬСтво. Случай $G=\mathrm{PSL}_{2}(q)$ разобран в [6], поэтому можем предполагать, что $G \neq \mathrm{PSL}_{2}(q)$. По лемме Шура имеем $C_{H}(G)=Z$. Предположим, что $A$ является нетривиальной абелевой нормальной подгруппой группы $H$. Рассмотрим $\chi$ как рациональный характер группы $H$. Тогда $\chi$ примитивен (см. лемму 2.3). Для любого простого делителя $r$ числа $|A|$ рассмотрим элементарную абелеву $r$-подгрупту $R$ такую, что $1 \neq R \subseteq A, R \triangleleft H$. Покажем, что $R=Z$. Действительно, по теореме Клиффорда [15] имеем $\left.\chi\right|_{R}=e \sum_{i=1}^{s} \rho_{i}$, где $e-$ целое положительное число и $\rho_{i}-$ различные неприводимые рациональные характеры группы $R$. Так как $\operatorname{deg} \chi$ нечетна, то $r=2$. Примитивность $\chi$ влечет $s=1$ и $\left.\chi\right|_{R}=e \rho_{1}$. Следовательно, $\rho_{1}$ является точньм неприводимьм рациональным характером элементарной абелевой 2-группы $R$. Поэтому $R=Z$. Это означает, что $A$ циклическая и $A=Z$.

ЛЕмма 3.2. Если $G \neq \mathrm{PSL}_{2}(7), m o \operatorname{sol}(H)=Z$.

ДокАЗАТЕЛЬСтво. Предположим, что $G \neq \mathrm{PSL}_{2}(7)$ и $R=\operatorname{sol}(H) \neq Z$. Pacсмотрим производный ряд

$$
R=R^{(1)} \supset R^{(2)} \supset \cdots \supset R^{(s)} \supset R^{(s+1)}=1,
$$

где $R^{(i+1)}=\left[R^{(i)}, R^{(i)}\right]$. Положим $N=R^{(s-1)}$. Тогда $N$ - нормальная подгруппа в $H, N^{\prime}=[N, N] \neq 1$ и $\left[N^{\prime}, N^{\prime}\right]=1$. Так как $N^{\prime}$ является абелевой нормальной подгруппой группы $H$, то по лемме 3.1 имеем $N^{\prime}=Z$. Следовательно, $N$ - неабелева нильпотентная подгруппа. Поэтому $N$ равна прямому произведению силовских подгрупп. Тогда $N$ является 2-группой, поскольку $\operatorname{abel}(H)=Z$. По теореме Клиффорда имеем $\left.\chi\right|_{N}=e \sum_{i=1}^{s} \rho_{i}$. Так как $\operatorname{deg} \chi$ нечетна, $\operatorname{deg} \rho_{i}$ одинаковы и $\operatorname{deg} \rho_{i}|| N \mid, \operatorname{to} \operatorname{deg} \rho_{i}=1$. Следовательно, $Z=N^{\prime}$ лежит в ядре всех $\rho_{i}$; получили противоречие.

Теперь мы переходим к вычислению групп автоморфизмов инвариантных решеток. Сначала рассмотрим случай, когда $H$ импримитивна, т.е. $G=\mathrm{PSL}_{2}(5)$, $\mathrm{PSL}_{2}(7), \mathrm{PSp}_{4}(3)$. Случай $G=\mathrm{PSL}_{2}(q)$ разобран в [6]. 
Лемма 3.3. Если $G=\operatorname{PSp}_{4}(3)$ и $H=\operatorname{Aut}(\Lambda)-$ импримитивная группа, то $H=\mathbb{Z}_{2} \times G$.

ДокАЗАТЕЛЬСтво. Пусть $\Omega=\left\{V_{i}, i=1, \ldots, t\right\}$ - система импримитивности для $H$, т.е. $V=V_{1}+\cdots+V_{t}$ и $H$ действует на $\Omega$. Пусть $\varphi: H \rightarrow S_{\Omega}$ - естественньй гомоморфизм. Тогда по лемме 3.2 имеем $\operatorname{ker} \varphi=Z$. Так как $G \hookrightarrow H$ и группа $G$ не имеет подгрупп индексов $81,9,3$, то $t=27$. Согласно [16] имеем, что $\operatorname{soc}(H / Z)$ изоморфен $\operatorname{PSp}_{4}(3)$ или $A_{27}$. Изоморфизм $\operatorname{soc}(H / Z) \cong A_{27}$ невозможен, поскольку нерасшепляемое расширение $2 A_{27}$ имеет точные представления только четной размерности (см. [17]) и группа $A_{27}$ не имеет неприводимых представлений размерности 27 (см. [18]). Поэтому $\operatorname{soc}(H / Z) \cong \mathrm{PSp}_{4}(3)$ и $H=\mathbb{Z}_{2} \times G$. Лемма доказана.

Таким образом, нам осталось рассмотреть примитивньй случай.

Лемма 3.4. Если $G \neq \mathrm{PSL}_{2}(7)$ и $H$ - примитивная группа, то цоколь $L=\operatorname{soc}(H / Z)$ группь $H / Z$ является неабелевой простой группой, содержащей zpynny $G$.

ДоКАЗАТЕльСТВо. Пусть $\bar{H}=H / Z$ и $\bar{M}$ - минимальная нормальная подгруппа группы $\bar{H}$. Так как $\operatorname{sol}(H)=Z$, то $\bar{M}$ неразрешима и $\bar{M}=\bar{L}_{1} \times \cdots \times \bar{L}_{k}$, $\bar{L}_{1} \cong \ldots \cong \bar{L}_{k} \cong L, L$ - неабелева простая группа. Обозначим через $M, L_{1}, \ldots, L_{k}$ полные прообразы $\bar{M}, \bar{L}_{1}, \ldots, \bar{L}_{k}$ в $H$. Тогда $M=L_{1} \circ \ldots \circ L_{k}$ (центральное произведение). Пусть $d=d(L)$ - наименьшая степень точного проективного комплексного представления группы $L$. Так как $H$ - примитивная группа, то $\left.\chi\right|_{M}=e \sigma$ для некоторого характера группы $M$. Сушествуют неприводимые характеры $\rho_{i}$ групा $L_{i}$ такие, что $\sigma\left(x_{1} \cdots x_{k}\right)=\rho_{1}\left(x_{1}\right) \cdots \rho_{k}\left(x_{k}\right)$ для всех $x_{i} \in L_{i}$. Поэтому $\left.\chi\right|_{L_{i}}=\frac{\operatorname{deg} \chi}{\operatorname{deg} \rho_{i}} \rho_{i}$. Следовательно, $\rho_{i}-$ точные и $\operatorname{deg} \chi \geqslant d^{k}$.

Группа $G$ вложена в $\bar{H}$. Покажем, что $G$ нормализует каждую подгруппу $\bar{L}_{i}$. Действительно, если $G$ переставляет $\bar{L}_{1}, \ldots, \bar{L}_{k}$, то $k \geqslant P(G)$, где $P(G)$ обозначает минимальную степень перестановочного представления группы $G$. Следовательно,

$$
\operatorname{deg} \chi \geqslant d^{P(G)}
$$

что невозможно согласно [9], [19]-[21].

Рассмотрим естественный гомоморфизм $\pi: G \rightarrow \operatorname{Aut}\left(\bar{L}_{i}\right)=\operatorname{Aut}(L)=L \times \operatorname{Out}(L)$. Если $\operatorname{ker} \pi=G$, то $G \subseteq C_{H}\left(L_{i}\right), L_{i} \subseteq C_{H}(G)$ и $L_{i} \subseteq Z$, что невозможно. Следовательно, $\operatorname{ker} \pi=1$ и $G \hookrightarrow L \times \operatorname{Out}(\bar{L})$. Так как $G$ совершенная группа и $\operatorname{Out}(L)$ разрешима согласно "гипотезе Шрейера", то $G \hookrightarrow L$. Покажем, что $k=1$. Действительно, пусть $G_{i} \leqslant L_{i}, G_{i} / Z \cong G$. Положим $S=G_{i} G$ и $\psi=\left.\chi\right|_{S}$. Имеем $\left.\psi\right|_{G_{i}}=m \tau$, где $\tau$ - неприводимый характер группы $G_{i}$. Но тогда по лемме 2.2 и [13] имеем $\operatorname{deg} \tau=\operatorname{deg} \chi$, что влечет $\operatorname{deg} \rho_{i}=\operatorname{deg} \chi$ и $k=1$.

Следовательно, всякая минимальная нормальная подгруппа $\bar{M}$ группы $\bar{H}$ является неабелевой простой группой, содержащей группу $G$. Ясно, что такая подгруппа единственна. Лемма доказана.

Используя классификацию конечных простых групп, изучим все возможности для $\operatorname{soc}(H / Z)$.

Лемма 3.5. Пусть $G \neq \operatorname{PSL}_{2}(q), p>2$. Тогда цоколь $L=\operatorname{soc}(H / Z)$ группьь $H / Z$ не может бъть спорадической простой группой. 
ДокаЗАТЕЛЬСтво. Если $G \neq \mathrm{PSL}_{2}(q)$, то $\operatorname{deg} \chi=p^{i} \geqslant p^{3}$. Но спорадические группы не имеют неприводимых проективных рациональных характеров степени $p^{i} \geqslant p^{3}$ (см. [13]).

ЛЕмма 3.6. Пусть $G \neq \mathrm{PSL}_{2}(7)$ и цоколь $L=\operatorname{soc}(H / Z)$ группьи $H / Z$ является знакопеременной группой $A_{m}$. Тогда $G=\mathrm{PSL}_{2}(q), \operatorname{PSU}_{3}(q),{ }^{2} B_{2}(q)$ или ${ }^{2} G_{2}(q), G$ действует дважды транзитивно на множестве из $m$ точек, $m=\operatorname{deg} \chi+1 u \operatorname{Aut}(\Lambda) \cong \mathbb{Z}_{2} \times S_{m}$.

ДокАЗАТЕЛЬСТво. Пусть $L=A_{m}$. Тогда для полного прообраза $\bar{L}$ группы $L$ в $H$ имеем $\bar{L}=Z \times A_{m}$, поскольку все точные представления группы $2 A_{m}$ имеют четную размерность [17] ( $2 A_{m}$ обозначает нерасщепляемое расширение группы $\left.A_{m}\right)$. Так как $G \hookrightarrow A_{m}$, то согласно [22] имеем следующие две возможности:

1) $G \geqslant A_{m-2}$, но это влечет $|G| \geqslant \frac{1}{2}(m-2) ! \geqslant \frac{1}{2}(P(G)-2)$ !, что невозможно согласно [9], [19]-[21];

2) $G$ - дважды транзитивная группа, действующая на множестве из $m$ элементов, и $\left.\chi\right|_{A_{m}}$ - характер, соответствуюший разбиению $(m-1,1)$ (о параметризации неприводимых характеров групп $A_{m}$ и $S_{m}$ разбиениями см. [23]). Из списка [24] дважды транзитивных групп с неабелевым цоколем получаем $G=\mathrm{PSL}_{2}(q)$, $\mathrm{PSU}_{3}(q),{ }^{2} B_{2}(q),{ }^{2} G_{2}(q)$. Классификация $A_{m}$-инвариантных решеток в $(m-1)$-мерном модуле приведена в [25], откуда следует, что $\operatorname{Aut}(\Lambda) \cong \mathbb{Z}_{2} \times S_{m}$.

Лемма 3.7. Пусть цоколь $L=\operatorname{soc}(H / Z)$ группь $H / Z$ является группой типа Ли характеристики р. Тогда $L=G$.

ДокАЗАТЕльство. Предположим, что $L \neq G$. Группа $L$ имеет неприводимый рациональный проективный характер степени $|G|_{p}$. Следовательно, по лемме 2.2 и [13] имеем $|L|_{p}=|G|_{p}$. По теореме Бореля-Титса [26] группа $G$ содержится в некоторой параболической подгруппе $P$ группы $L$. Но это невозможно, поскольку $G$ простая. Лемма доказана.

Напомним некоторые обозначения. Для простой группы $L$ типа Ли над полем $\mathbb{F}_{q}$ число $R_{p}(L)$ обозначает наименьшую степень нетривиальных проективных характеров над полем характеристики $p$ и $R_{p^{\prime}}(L)$ обозначает наименьшую степень нетривиальных проективных характеров над полем характеристики, отличной от $p$. В дальнейшем будем использовать значения $R_{p}(L)$ из [9] и нижние границы для $R_{p^{\prime}}(L)$ из [27] и [28] без дополнительных ссылок. Следующий результат является следствием из [28].

ЛЕмма 3.8. Пусть $L$ - классическая простая группа размерности $n$ над полем $\mathbb{F}_{q}, q=p^{f}$.

1) $\operatorname{Ecлu~} n \geqslant 6$, mo $R_{p^{\prime}}(L) \geqslant \frac{1}{2}\left(q^{n / 2}-1\right)$.

2) Если $n \geqslant 8$ и $p=2$, то $R_{p^{\prime}}(L) \geqslant \frac{1}{2} q^{n-3}$, за исключением $R_{p^{\prime}}\left(\Omega_{8}^{+}(2)\right)=8$.

Лемма 3.9. Пусть $G=L_{n}(q), n \geqslant 3, p>2$. Тогда иоколь $L=\operatorname{soc}(H / Z)$ зруппь $H / Z$ не может бъть әруппой типа Ли характеристики, отличной om $p$.

ДокАЗАтЕльство. 1) Предположим, что $L$ - классическая простая группа размерности $k$ над $\mathbb{F}_{s}, s=r^{t}, r$ простое, $r \neq p$. Тогда

$$
k=R_{r}(L) \geqslant R_{p^{\prime}}(G) \geqslant \frac{q^{n}-1}{q-1}-n \geqslant q^{n-1}+1 \geqslant 10 .
$$


Далее, если $r \neq 2$, то $s \geqslant 3$ и

$$
\begin{aligned}
R_{r^{\prime}}(L) & \geqslant \frac{1}{2}\left(s^{k / 2}-1\right) \geqslant \frac{1}{2}\left(s^{\left(q^{n-1}+1\right) / 2}-1\right) \geqslant \frac{1}{2}\left(3^{\left(q^{n-1}+1\right) / 2}-1\right) \\
& >q^{n(n-1) / 2}=\operatorname{deg} \chi .
\end{aligned}
$$

Пусть $r=2$. Тогда $G \neq \Omega_{8}^{+}(2)$, поскольку $\Omega_{8}^{+}(2)$ не имеет неприводимых проективных представлений степени $p^{i}, p>2$. Поэтому

$$
R_{r^{\prime}}(L) \geqslant \frac{1}{2} s^{k-3} \geqslant \frac{1}{2} s^{q^{n-1}-3} \geqslant 2^{q^{n-1}-4}>q^{n(n-1) / 2}=\operatorname{deg} \chi .
$$

2) Предположим, что $L$ - исключительная простая группа типа Ли над $\mathbb{F}_{s}$, $s=r^{t}, r \neq p$. Тогда

$$
R_{r}(L) \geqslant R_{p^{\prime}}(G) \geqslant \frac{q^{n}-1}{q-1}-n .
$$

Если $n=3, q=3$, то $R_{r}(L) \geqslant 10$ и $L=E_{6}(s), E_{7}(s), E_{8}(s),{ }^{2} E_{6}(s), F_{4}(s)$, ${ }^{2} F_{4}(s)$ или ${ }^{2} F_{4}(2)^{\prime}$. Но группа Титса ${ }^{2} F_{4}(2)^{\prime}$ не имеет проективных рациональных представлений размерности $q^{n(n-1) / 2}=\operatorname{deg} \chi=27$, и для остальных групп имеем

$$
R_{r^{\prime}}(L) \geqslant 44>27=q^{n(n-1) / 2}=\operatorname{deg} \chi,
$$

что невозможно. Если $n=3,5 \leqslant q \leqslant 13$, то $R_{r}(L) \geqslant 28$ и $L=E_{7}(s), E_{8}(s)$, но тогда

$$
R_{r^{\prime}}(L) \geqslant s^{15}\left(s^{2}-1\right) \geqslant 2^{16}>q^{3}=\operatorname{deg} \chi .
$$

Если $n=4,3 \leqslant q \leqslant 5$, то $R_{r}(L) \geqslant 36, L=E_{7}(s), E_{8}(s)$ и

$$
R_{r^{\prime}}(L) \geqslant s^{15}\left(s^{2}-1\right) \geqslant 2^{16}>q^{6}=\operatorname{deg} \chi .
$$

Если $n=5, q=3$, то $R_{r}(L) \geqslant 116, L=E_{8}(s)$ и

$$
R_{r^{\prime}}(L) \geqslant s^{27}\left(s^{2}-1\right) \geqslant 2^{28}>q^{10}=\operatorname{deg} \chi .
$$

В оставшихся случаях (т.е. при $n=3, q \geqslant 17$; или $n=4, q \geqslant 7$; или $n=5, q \geqslant 5$; или $n>5)$ имеем

$$
R_{r}(L)>248
$$

что невозможно.

Лемма 3.10. Пусть $G=\operatorname{PSU}_{n}(q), n \geqslant 3, p>2$, цоколь $L=\operatorname{soc}(H / Z)$ группь $H / Z$ является группой типа Ли характеристики, отличной от $p$. Тогда $G=\operatorname{PSU}_{3}(3)$ u $L=\operatorname{Sp}_{6}(2)$.

ДокАЗАТЕЛЬСтво. 1) Предположим, что $L-$ классическая простая группа размерности $k$ над $\mathbb{F}_{s}, s=r^{t}, r$ простое, $r \neq p$. Тогда

$$
k=R_{r}(L) \geqslant R_{p^{\prime}}(G) \geqslant \frac{q^{n}-q}{q+1} \geqslant 6 .
$$

Пусть $(q, n) \neq(3,3)$. Если $r \neq 2$, то $s \geqslant 3$ и

$$
R_{r^{\prime}}(L) \geqslant \frac{1}{2}\left(s^{k / 2}-1\right) \geqslant \frac{1}{2}\left(3^{\left(q^{n}-q\right) /(2(q+1))}-1\right)>q^{n(n-1) / 2}=\operatorname{deg} \chi .
$$


Если $r=2$, то $G \neq \Omega_{8}^{+}(2)$, поскольку $\Omega_{8}^{+}(2)$ не имеет неприводимых проективных представлений степени $p^{i}, p>2$. Поэтому

$$
R_{r^{\prime}}(L) \geqslant \frac{1}{2} s^{k-3} \geqslant 2^{\left(q^{n}-q\right) /(q+1)-4}>q^{n(n-1) / 2}=\operatorname{deg} \chi .
$$

Осталось рассмотреть случай $q=n=3$.

1. $L=L_{k}(s)$. Тогда $R_{r^{\prime}}(L) \geqslant s^{k-1}-1 \geqslant 2^{5}-1>q^{3}=\operatorname{deg} \chi$.

2. $L=\operatorname{PSU}_{k}(s)$. Тогда $(k, s) \neq(6,2)$, поскольку $\operatorname{PSU}_{6}(2)$ не имеет неприводимых проективных характеров степени 27 , и поэтому

$$
R_{r^{\prime}}(L) \geqslant \frac{s^{k}-s}{s+1}>3^{3}=\operatorname{deg} \chi .
$$

3. $L=\mathrm{PSp}_{2 m}(s)$. Если $s$ нечетно, то $(2 m, s) \neq(6,3)$ (так как $\mathrm{PSp}_{6}(3)$ не имеет неприводимых проективных характеров степени 27$)$ и

$$
R_{r^{\prime}}(L) \geqslant \frac{s^{m}-1}{2}>3^{3}=\operatorname{deg} \chi .
$$

Если $s$ четно и $(2 m, s) \neq(6,2)$, то

$$
R_{r^{\prime}}(L) \geqslant \frac{\left(s^{m}-1\right)\left(s^{m}-s\right)}{2(s+1)}>3^{3}=\operatorname{deg} \chi .
$$

Случай $L=\operatorname{Sp}_{6}(2)$ возможен, поскольку $\operatorname{PSU}_{3}(3) \hookrightarrow \operatorname{Sp}_{6}(2)$ и группы $\operatorname{PSU}_{3}(3)$, $\mathrm{Sp}_{6}(2)$ действуют дважды транзитивно на множестве из 28 элементов.

4. $L=\mathrm{P} \Omega_{2 m+1}(s), m \geqslant 3$. Тогда

$$
R_{r^{\prime}}(L) \geqslant s^{m-1}\left(s^{m-1}-1\right) \geqslant 3^{2}\left(3^{2}-1\right)>3^{3}=\operatorname{deg} \chi .
$$

5. $L=\mathrm{P} \Omega_{2 m}^{+}(s), m \geqslant 4$. Тогда $(m, s) \neq(4,2)$ (так как $\mathrm{P} \Omega_{8}^{+}(2)$ не имеет неприводимых проективных характеров степени 27$)$ и

$$
R_{r^{\prime}}(L) \geqslant s^{m-2}\left(s^{m-1}-1\right)>3^{3}=\operatorname{deg} \chi .
$$

6. $L=\mathrm{P} \Omega_{2 m}^{-}(s), m \geqslant 4$. Тогда

$$
R_{r^{\prime}}(L) \geqslant \frac{\left(s^{m}+1\right)\left(s^{m-1}-s\right)}{s^{2}-1}-m+2>3^{3}=\operatorname{deg} \chi .
$$

2) Предположим, что $L$ - исключительная простая группа типа Ли над $\mathbb{F}_{s}$, $s=r^{t}, r$ простое, $r \neq p$. Тогда

$$
R_{r}(L) \geqslant R_{p^{\prime}}(G) \geqslant \frac{q^{n}-q}{q+1} .
$$

Если $n=3, q=3$, то $R_{r}(L) \geqslant 6$, поэтому или $R_{r^{\prime}}(L)>3^{3}$, или $L=G_{2}(4)$, ${ }^{3} D_{4}(2),{ }^{2} F_{4}(2)^{\prime},{ }^{2} B_{2}(8)$, но последние группы не имеют неприводимых проективных рациональных характеров степени 27.

Если $n=3, q=5$, то $R_{r}(L) \geqslant 20$, поэтому или $R_{r^{\prime}}(L)>5^{3}$, или $L=F_{4}(2)$, ${ }^{2} F_{4}(2)^{\prime}$, но последние группы не имеют неприводимых проективных характеров степени $5^{3}$.

Если $n=3,7 \leqslant q \leqslant 13$, то $R_{r}(L) \geqslant 42$, поэтому $L=E_{7}(s), E_{8}(s)$ и $R_{r^{\prime}}(L) \geqslant$ $s^{15}\left(s^{2}-1\right) \geqslant 2^{16}>q^{3}=\operatorname{deg} \chi$.

Если $n=4, q=3$, то $R_{r}(L) \geqslant 20$, поэтому или $R_{r^{\prime}}(L)>q^{6}=\operatorname{deg} \chi$, или $L=F_{4}(2),{ }^{2} F_{4}(2)^{\prime}$, но эти группы не имеют неприводимых проективных характеров степени $3^{6}$.

Если $n=4, q=5$, то $R_{r}(L) \geqslant 104, L=E_{8}(s), R_{r^{\prime}}(L) \geqslant s^{27}\left(s^{2}-1\right)>5^{6}=$ $\operatorname{deg} \chi$.

Если $n=5, q=3$, то $R_{r}(L) \geqslant 60, L=E_{8}(s), R_{r^{\prime}}(L) \geqslant s^{27}\left(s^{2}-1\right)>3^{10}=\operatorname{deg} \chi$.

В оставшихся случаях (т.е. при $n=3, q \geqslant 17$; или $n=4, q \geqslant 7$; или $n=5$, $q \geqslant 5$; или $n>5)$ имеем $R_{r}(L)>248$, что невозможно. 
Лемма 3.11. Пусть $G=\operatorname{PSp}_{2 m}(q), m \geqslant 2, p>2$. Тогда иоколь $L=$ $\operatorname{soc}(H / Z)$ группы $H / Z$ не может быть группой типа Ли характеристики, отличной от $p$.

ДокАЗАтЕльство. 1) Предположим, что $L$ - классическая простая группа размерности $k$ над $\mathbb{F}_{s}, s=r^{t}, r$ простое, $r \neq p$. Пусть $(m, q) \neq(2,3),(2,5),(3,3)$. Тогда

$$
k=R_{r}(L) \geqslant R_{p^{\prime}}(G) \geqslant \frac{q^{m}-1}{2}>10 .
$$

Если $r \neq 2$, то $s \geqslant 3$ и

$$
R_{r^{\prime}}(L) \geqslant \frac{1}{2}\left(s^{k / 2}-1\right) \geqslant \frac{1}{2}\left(3^{\left(q^{m}-1\right) / 4}-1\right)>q^{m^{2}}=\operatorname{deg} \chi .
$$

Если $r=2$, то $G \neq \Omega_{8}^{+}(2)$, поскольку $\Omega_{8}^{+}(2)$ не имеет неприводимых проективных представлений степени $p^{i}, p>2$. Поэтому

$$
R_{r^{\prime}}(L) \geqslant \frac{1}{2} s^{k-3} \geqslant 2^{\left(q^{m}-1\right) / 2-4}>q^{m^{2}}=\operatorname{deg} \chi .
$$

Следовательно, нам надо рассмотреть случаи $(m, q)=(2,3),(2,5),(3,3)$.

1. $L=L_{k}(s)$. Тогда

$$
R_{r^{\prime}}(L) \geqslant \frac{s^{k}-1}{s-1}-k>q^{m^{2}}=\operatorname{deg} \chi,
$$

кроме случаев $m=2, q=3, s=2, k=4,5,6 ; m=2, q=3, s=4, k=4$; $m=3, q=3, s=2, k=13,14$ (мы использовали то, что $q$ и $s$ взаимно просты и $\left.k \geqslant\left(q^{m}-1\right) / 2\right)$. Но группы $L_{4}(2), L_{5}(2)$ не обладают неприводимыми характерами степени $3^{4}$; невозможность остальных случаев следует из значений первых двух степеней характеров групп $\mathrm{PSL}_{k}(s)$ (см. [29]).

2. $L=\mathrm{PSU}_{k}(s)$. Тогда

$$
R_{r^{\prime}}(L) \geqslant \frac{s^{k}-s}{s+1}>q^{m^{2}}=\operatorname{deg} \chi
$$

кроме случаев $m=2, q=3, s=2, k=4,5,6,7 ; m=2, q=3, s=4, k=4$; $m=3, q=3, s=2, k=13,14,15$. $\mathrm{Ho} \mathrm{PSU}_{4}(2) \cong \operatorname{PSp}_{4}(3)$, групшы $\operatorname{PSU}_{5}(2)$ и $\mathrm{PSU}_{6}(2)$ не имеют неприводимых комплексных характеров степени 81. Невозможность остальных случаев следует из значений первых трех степеней характеров группы $\mathrm{PSU}_{k}(s)$ (см. [29]).

3. $L=\operatorname{PSp}_{2 d}(s)$. Тогда $2 d=R_{r}(L) \geqslant R_{p^{\prime}}(G) \geqslant\left(q^{m}-1\right) / 2$.

Если $s$ нечетно, то $R_{r^{\prime}}(L) \geqslant\left(s^{d}-1\right) / 2>q^{m^{2}}=\operatorname{deg} \chi$, кроме случаев $m=2$, $q=3, s=5, d=2,3 ; m=2, q=3, s=7, d=2 ; m=2, q=3, s=11, d=2 ; m=2$, $q=5, s=3, d=6$. Первые три степени нетривиальных комплексных характеров группы $\operatorname{PSp}_{2 d}(s)$ равны $\frac{s^{d} \pm 1}{2}$ и $\frac{\left(s^{d}-1\right)\left(s^{d}-s\right)}{2(s+1)}$ (см. [29]), поэтому $\operatorname{PSp}_{6}(5)$ не имеет характеров степени 81. Далее, группы $\mathrm{PSp}_{4}(5), \mathrm{PSp}_{4}(7), \mathrm{PSp}_{4}(11)$ не имеют характеров степени 81, поскольку 81 не делит порядки этих групп. Аналогично, $\mathrm{PSp}_{12}(3)$ не имеет характеров степени $5^{4}$.

Если $s$ четно, то

$$
R_{r^{\prime}}(L) \geqslant \frac{\left(s^{d}-1\right)\left(s^{d}-s\right)}{2(s+1)}
$$


за исключением $R_{r^{\prime}}\left(\operatorname{Sp}_{4}(2)^{\prime}\right)=2$. Следовательно, $R_{r^{\prime}}(L)>q^{m^{2}}$, кроме случаев $m=2, q=3, s=2, d=2,3,4 ; m=2, q=3, s=4, d=2 ; m=3, q=3$, $s=2, d=7,8$. Группы $\mathrm{Sp}_{4}(2)^{\prime}, \mathrm{Sp}_{6}(2), \mathrm{Sp}_{8}(2), \mathrm{Sp}_{4}(4)$ не имеют характеров степени 81 (см. [13]), и групшы $\mathrm{Sp}_{14}(2), \mathrm{Sp}_{16}(2)$ не имеют неприводимых характеров степени $3^{9}$ (если такой характер $\psi$ существует, то, используя сведения об индексе централизатора полупростого элемента в $\mathrm{Sp}_{2 d}(2)$ из [29], можно показать, что $\psi$ - унипотентньй характер; далее, методом доказательства леммы 2.2 легко убедиться в том, что групшы $\mathrm{Sp}_{14}(2), \mathrm{Sp}_{16}(2)$ не имеют унипотентных неприводимых характеров степени $3^{9}$ ).

4. $L=\mathrm{P} \Omega_{2 d+1}(s), d \geqslant 3, s=r^{t}$. Тогда

$$
2 d+1=R_{r}(L) \geqslant R_{p^{\prime}}(G) \geqslant \frac{q^{m}-1}{2}
$$

и для наших значений $m$ и $q$ имеем

$$
R_{r^{\prime}}(L) \geqslant s^{d-1}\left(s^{d-1}-1\right)>q^{m^{2}}
$$

5. $L=\mathrm{P} \Omega_{2 d}^{ \pm}(s), d \geqslant 4, s=r^{t}$. Тогда

$$
2 d=R_{r}(L) \geqslant R_{p^{\prime}}(G) \geqslant \frac{q^{m}-1}{2}, \quad R_{r^{\prime}}(L) \geqslant\left(s^{d-1}+1\right)\left(s^{d-2}-1\right)>q^{m^{2}} .
$$

2) Предположим, что $L$ - исключительная простая группа типа Ли над $\mathbb{F}_{s}$, $s=r^{t}, r$ простое, $r \neq p$. Тогда

$$
R_{r}(L) \geqslant R_{p^{\prime}}(G) \geqslant \frac{q^{m}-1}{2} .
$$

Если $m=2, q=3$, то или $R_{r^{\prime}}(L)>3^{4}$, или $L=G_{2}(4),{ }^{3} D_{4}(2),{ }^{2} F_{4}(2){ }^{\prime},{ }^{2} B_{2}(8)$, но последние группы не имеют неприводимых проективных характеров степени $3^{4}$.

Если $m=2, q=5$, то $R_{r}(L) \geqslant 12, L=F_{4}(s),{ }^{2} F_{4}(s){ }^{\prime}, E_{6}(s),{ }^{2} E_{6}(s), E_{7}(s)$, $E_{8}(s)$, поэтому или $R_{r^{\prime}}(L)>5^{4}$, или $L=F_{4}(2),{ }^{2} F_{4}(2)^{\prime}$, но последние групшы не имеют неприводимых проективных характеров степени $5^{4}$.

Если $m=2, q=7$, то $R_{r}(L) \geqslant 24, L=F_{4}(s),{ }^{2} F_{4}(s){ }^{\prime}, E_{6}(s),{ }^{2} E_{6}(s), E_{7}(s)$, $E_{8}(s)$, поэтому или $R_{r^{\prime}}(L)>7^{4}$, или $L=F_{4}(2),{ }^{2} F_{4}(2)^{\prime}, E_{6}(2),{ }^{2} E_{6}(2)$, но последние группы не имеют неприводимых проективных характеров степени $7^{4}$.

Если $m=2,9 \leqslant q \leqslant 19$, то $R_{r}(L) \geqslant 40, L=E_{7}(s), E_{8}(s)$, поэтому $R_{r^{\prime}}(L)>19^{4}$.

Если $m=3, q=3$, то $R_{r}(L) \geqslant 13, L=F_{4}(s),{ }^{2} F_{4}(s){ }^{\prime}, E_{6}(s),{ }^{2} E_{6}(s), E_{7}(s)$, $E_{8}(s)$, поэтому или $R_{r^{\prime}}(L)>3^{9}$, или $L=F_{4}(2),{ }^{2} F_{4}(2)^{\prime}, E_{6}(2),{ }^{2} E_{6}(2)$, но последние групшы не имеют неприводимых проективных характеров степени $7^{4}$.

Если $m=3, q=5,7$, то $R_{r}(L) \geqslant 62, L=E_{8}(s)$, поэтому $R_{r^{\prime}}(L)>7^{9}$.

Если $m=4, q=3$, то $R_{r}(L) \geqslant 40, L=E_{7}(s), E_{8}(s)$, поэтому или $R_{r^{\prime}}(L)>3^{16}$, или $L=E_{7}(2)$, но эта группа не имеет неприводимых проективных характеров степени $3^{16}$.

Если $m=5, q=3$, то $R_{r}(L) \geqslant 121, L=E_{8}(s)$, поэтому или $R_{r^{\prime}}(L)>3^{25}$, или $L=E_{8}(2)$, но эта группа не имеет неприводимых проективных характеров степени $3^{25}$.

В оставшихся случаях (т.е. при $m=2, q>19$; или $m=3, q>7$; или $m=4$, $q>3$; или $m=5, q>3$; или $m>5$ ) имеем $R_{r}(L)>248$, что невозможно. 
Лемма 3.12. Пусть $G=\mathrm{P} \Omega_{2 m}^{ \pm}(q), m \geqslant 4, p>2$. Тогда цоколь $L=$ $\operatorname{soc}(H / Z)$ группы $H / Z$ не может быть группой типа Ли характеристики, отличной от $p$.

ДокАЗАтЕльство. 1) Предположим, что $L$ - классическая простая группа размерности $k$ над $\mathbb{F}_{s}, s=r^{t}, r$ простое, $r \neq p$. Тогда

$$
\begin{aligned}
k & =R_{r}(L) \geqslant R_{p^{\prime}}(G) \geqslant\left(q^{m-1}+1\right)\left(q^{m-2}-1\right), \\
R_{r^{\prime}}(L) & \geqslant \frac{1}{2}\left(s^{k / 2}-1\right) \geqslant \frac{1}{2}\left(2^{q^{m-1}}-1\right)>q^{m(m-1)}=\operatorname{deg} \chi .
\end{aligned}
$$

2) Предположим, что $L$ - исключительная простая группа типа Ли над $\mathbb{F}_{s}$, $s=r^{t}, r \neq p$. Тогда

$$
R_{r}(L) \geqslant R_{p^{\prime}}(G) \geqslant\left(q^{m-1}+1\right)\left(q^{m-2}-1\right)>248,
$$

кроме случая $m=4, q=3$. Но тогда $R_{r}(L) \geqslant\left(3^{3}+1\right)\left(3^{2}-1\right)=224$ и $L=E_{8}(s)$. Поэтому

$$
R_{r^{\prime}}(L) \geqslant s^{27}\left(s^{2}-1\right)>q^{m(m-1)}=\operatorname{deg} \chi .
$$

Лемма 3.13. Пусть $G=\mathrm{P} \Omega_{2 m+1}(q), m \geqslant 3, p>2$. Тогда иоколь $L=$ $\operatorname{soc}(H / Z)$ группы $H / Z$ не может быть группой типа Ли характеристики, отличной от $p$.

ДокАЗАтЕльство. 1) Предположим, что $L$ - классическая простая группа размерности $k$ над $\mathbb{F}_{s}, s=r^{t}, r$ простое, $r \neq p$. Тогда

$$
\begin{gathered}
k=R_{r}(L) \geqslant R_{p^{\prime}}(G) \geqslant q^{m-1}\left(q^{m-1}-1\right), \\
R_{r^{\prime}}(L) \geqslant \frac{1}{2}\left(s^{k / 2}-1\right) \geqslant \frac{1}{2}\left(s^{q^{m-1}\left(q^{m-1}-1\right) / 2}-1\right)>q^{m^{2}}=\operatorname{deg} \chi .
\end{gathered}
$$

2) Предположим, что $L$ - исключительная простая группа типа Ли над $\mathbb{F}_{s}$, $s=r^{t}, r \neq p$. Тогда

$$
R_{r}(L) \geqslant R_{p^{\prime}}(G) \geqslant q^{m-1}\left(q^{m-1}-1\right)>248,
$$

кроме случая $m=q=3$. Но тогда $R_{r}(L) \geqslant 3^{2}\left(3^{2}-1\right)=72$ и $L=E_{8}(s)$. Поэтому

$$
R_{r^{\prime}}(L) \geqslant s^{27}\left(s^{2}-1\right)>3^{9}=q^{m^{2}}=\operatorname{deg} \chi .
$$

Лемма 3.14. Пусть $G$ - әрупna muna $E_{6}(q),{ }^{2} E_{6}(q), E_{7}(q), E_{8}(q), E_{4}(q)$. Тогда цоколь $L=\operatorname{soc}(H / Z)$ группь $H / Z$ не может бъть группой типа Ли характеристики, отличной от р.

ДоказАТЕльство. 1) Предположим, что $L$ - классическая простая группа размерности $k$ над $\mathbb{F}_{s}, s=r^{t}, r$ простое, $r \neq p$. Тогда

$$
\begin{gathered}
k=R_{r}(L) \geqslant R_{p^{\prime}}(G) \geqslant q^{6}\left(q^{2}-1\right), \\
R_{r^{\prime}}(L) \geqslant \frac{1}{2}\left(s^{k / 2}-1\right) \geqslant \frac{1}{2}\left(s^{q^{6}\left(q^{2}-1\right) / 2}-1\right)>q^{120} \geqslant \operatorname{deg} \chi .
\end{gathered}
$$

2) Предположим, что $L$ - исключительная простая группа типа Ли над $\mathbb{F}_{s}$, $s=r^{t}, r \neq p$. Тогда

$$
R_{r}(L) \geqslant R_{p^{\prime}}(G) \geqslant q^{6}\left(q^{2}-1\right)>248,
$$

получили противоречие. 
Лемма 3.15. Пусть $G={ }^{2} G_{2}(q), q=p^{f} \geqslant p^{3}, p=3$. Тогда иоколь $L=$ $\operatorname{soc}(H / Z)$ группь $H / Z$ не может быть группой типа Ли характеристики, отличной от $p$.

ДокаЗАТЕльство. 1) Предположим, что $L$ - классическая простая группа размерности $k$ над $\mathbb{F}_{s}, s=r^{t}, r$ простое, $r \neq p$. Тогда

$$
\begin{gathered}
k=R_{r}(L) \geqslant R_{p^{\prime}}(G) \geqslant q(q-1), \\
R_{r^{\prime}}(L) \geqslant \frac{1}{2}\left(s^{k / 2}-1\right) \geqslant \frac{1}{2}\left(s^{q(q-1) / 2}-1\right)>q^{3}=\operatorname{deg} \chi .
\end{gathered}
$$

2) Предположим, что $L$ - исключительная простая группа типа Ли над $\mathbb{F}_{s}$, $s=r^{t}, r \neq p$. Тогда

$$
R_{r}(L) \geqslant R_{p^{\prime}}(G) \geqslant q(q-1)>248,
$$

получили противоречие.

Лемма 3.16. Пусть $G={ }^{3} D_{4}(q), p>2$. Тогда цоколь $L=\operatorname{soc}(H / Z)$ группьь $H / Z$ не может бъть группой типа Ли характеристики, отличной от $p$.

ДокАЗАТЕЛЬство. 1) Предположим, что $L$ - классическая простая группа размерности $k$ над $\mathbb{F}_{s}, s=r^{t}, r$ простое, $r \neq p$. Тогда

$$
\begin{gathered}
k=R_{r}(L) \geqslant R_{p^{\prime}}(G) \geqslant q^{3}\left(q^{2}-1\right), \\
R_{r^{\prime}}(L) \geqslant \frac{1}{2}\left(s^{k / 2}-1\right) \geqslant \frac{1}{2}\left(s^{q^{3}\left(q^{2}-1\right) / 2}-1\right)>q^{12}=\operatorname{deg} \chi .
\end{gathered}
$$

2) Предположим, что $L$ - исключительная простая группа типа Ли над $\mathbb{F}_{s}$, $s=r^{t}, r \neq p$. Тогда

$$
R_{r}(L) \geqslant R_{p^{\prime}}(G) \geqslant q^{3}\left(q^{2}-1\right)>248,
$$

кроме $q=3$. Но в этом случае имеем $R_{r}(L)>56, L=E_{8}(s), R_{r^{\prime}}(L) \geqslant s^{27}\left(s^{2}-1\right)$ $>3^{3}=\operatorname{deg} \chi$.

Лемма 3.17. Пусть $G=G_{2}(q), p>2$. Тогда цоколь $L=\operatorname{soc}(H / Z)$ группьь $H / Z$ не может быть группой типа Ли характеристики, отличной от $p$.

ДокАЗАТЕльство. 1) Предположим, что $L$ - классическая простая группа размерности $k$ над $\mathbb{F}_{s}, s=r^{t}, r$ простое, $r \neq p$. Если $q \neq 3$, то

$$
\begin{gathered}
k=R_{r}(L) \geqslant R_{p^{\prime}}(G) \geqslant q\left(q^{2}-1\right), \\
R_{r^{\prime}}(L) \geqslant \frac{1}{2}\left(s^{k / 2}-1\right) \geqslant \frac{1}{2}\left(s^{q\left(q^{2}-1\right) / 2}-1\right)>q^{6}=\operatorname{deg} \chi .
\end{gathered}
$$

Если $q=3$, то $k=R_{r}(L) \geqslant R_{p^{\prime}}(G)=14$. При нечетных $s$ имеем

$$
R_{r^{\prime}}(L) \geqslant \frac{1}{2}\left(s^{k / 2}-1\right) \geqslant \frac{1}{2}\left(5^{7}-1\right)>q^{6}=\operatorname{deg} \chi .
$$

При четных $s$ имеем

$$
R_{r^{\prime}}(L) \geqslant \frac{1}{2} s^{k-3} \geqslant 2^{10}>q^{6}=\operatorname{deg} \chi .
$$

2) Предположим, что $L$ - исключительная простая группа типа Ли над $\mathbb{F}_{s}$, $s=r^{t}, r \neq p$. Пусть $q>3$. Тогда

$$
R_{r}(L) \geqslant R_{p^{\prime}}(G) \geqslant q\left(q^{2}-1\right)>248,
$$


кроме случая $q=5$. Но в этом случае имеем $R_{r}(L)>120, L=E_{8}(s), R_{r^{\prime}}(L) \geqslant$ $s^{27}\left(s^{2}-1\right)>5^{6}=\operatorname{deg} \chi$.

Осталось рассмотреть случай $q=3$. Так как $R_{r}(L) \geqslant R_{p^{\prime}}(G)=14$, то $L=$ $F_{4}(s), E_{6}(s),{ }^{2} E_{6}(s), E_{7}(s), E_{8}(s),{ }^{2} F_{4}(s),{ }^{2} F_{4}(2){ }^{\prime}$. Если $L=E_{6}(s),{ }^{2} E_{6}(s), E_{7}(s)$, $E_{8}(s)$, то $R_{r^{\prime}}(L) \geqslant s^{9}\left(s^{2}-1\right)>3^{6}=\operatorname{deg} \chi$. Пусть $L=F_{4}(s)$. Группа $F_{4}(2)$ не имеет неприводимых характеров степени $3^{6}$. При $s>2$ имеем $R_{r^{\prime}}(L)>s^{6} \geqslant 3^{6}=$ $\operatorname{deg} \chi$. Пусть $L={ }^{2} F_{4}(s), s \geqslant 8$. Тогда $R_{r^{\prime}}(L) \geqslant s^{4}(s-1) \sqrt{s / 2}>3^{6}=\operatorname{deg} \chi$. Наконец, группа $L={ }^{2} F_{4}(2)^{\prime}$ не имеет неприводимых характеров степени $3^{6}$.

ДОКАЗАТЕЛЬСТВо ТЕОРЕмЫ. При $G=\mathrm{PSL}_{2}(q)$ утверждение теоремы следует из [6]. Случай импримитивного $H$ рассмотрен в лемме 3.3. Наконец, леммы 3.4-3.17 доказывают теорему для примитивного $H$.

\section{Список литературы}

1. Иванов Д. Н. Ортогональные разложения алгебр Ли типа $A_{p^{n}-1}$ и $D_{n}$ с конечным числом классов подобных инвариантных подрешеток // Вестн. МГУ. Сер. 1. Матем., мех. 1989. № 2. С. 40-43.

2. Абдухаликов $K . C$. Целочисленные инвариантные решетки в алгебрах Ли типа $A_{p^{m}}-1$ // Матем. сб. 1993. Т. 184. № 4. С. 61-104.

3. Абдухаликов K. С. Целочисленные решетки, ассоциированные с конечной афффинной группой // Матем. сб. 1994. Т. 185. № 12. С. 3-18.

4. Бондал А.И., Кострикин А.И., Фам Хыу Тьеп. Инвариантные решетки, решетка Лича и ее четные унимодулярные аналоги в алгебрах $A_{p-1} / /$ Матем. сб. 1986. T. 130 (172). № 4 (8). C. 435-464.

5. Kostrikin A. I., Pham Huu Tiep. Orthogonal decompositions and integral lattices. Berlin: Walter de Gruyter, 1994.

6. Буриченко В. П. Инвариантные решетки в модуле Стейнберга и их групшы изометрий // Матем. сб. 1993. Т. 184. № 12. С. 145-156.

7. Carter $R$. W. Finite groups of Lie type. Conjugacy classes and complex characters. New York: Wiley and Sons, 1985.

8. Zsigmondy K. Zur Theorie der Potenzreste // Monatsh. Math. Phys. 1892. V. 3. P. 265-284.

9. Kleidman P.B., Liebeck M.W. The subgroup structure of the finite classical groups. Cambridge: Cambridge Univ. Press 1990.

10. Lusztig G. Characters of reductive groups over a finite field. Princeton: Princeton Univ. Press, 1984.

11. Lusztig G. On the representations of reductive groups with disconnected center // Astérisque. 1988. V. 168. P. 157-166.

12. Digne F., Michel J. Representations of finite groups of Lie type. Cambridge: Cambridge Univ. Press, 1991.

13. Conway J.H., Curtis R.T., Norton S.P., Parker R.A., Wilson R.A. Atlas of finite groups. Oxford: Clarendon Press, 1985.

14. Guralnick R. M. Subgroups of prime power index in a simple group // J. Algebra. 1983. V. 81. P. 304-311.

15. Кәртис Ч., Райнер И. Теория представлений конечных групп и ассоциативных алгебр. M.: Наука, 1969.

16. Dixon J., Mortimer B. The primitive permutation groups of degree less than $1000 / /$ Math. Proc. Cambridge Philos. Soc. 1988. V. 103. P. 213-238.

17. Wagner $A$. An observation on the degrees of projective representations of the symmetric and alternating groups over an arbitrary field // Arch. Math. 1977. V. 29. P. 583-589.

18. Rasala $R$. On the minimal degrees of characteres of $S_{n} / / \quad$ J. Algebra. 1977. V. 45. P. $132-181$.

19. Cooperstein B. N. Minimal degree for a permutation representation of a classical group // Israel J. Math. 1978. V. 30. P. 213-235.

20. Liebeck M. W., Saxl J. On the orders of maximal subgroups of the finite exceptional groups of Lie type // Proc. London Math. Soc. (3) 1987. V. 55. P. 299-330. 
21. Liebeck $M$. W., Saxl J. Maximal subgroups of finite simple groups and their automorphism groups // Contemp. Math. 1992. V. 131. P. 243-259.

22. Saxl J. The complex caharacters of the symmetric groups that remains irreducible in subgroups // J. Algebra. 1987. V. 111. P. 210-219.

23. James G.D. The representation theory of the symmetric groups. Berlin: Springer-Verlag, 1978.

24. Cameron P. J. Finite permutation groups and finite simple groups // Bull. London Math. Soc. 1981. V. 13. P. 1-22.

25. Plesken $W$. On absolutely irreducible representations of orders // Number Theory and Algebra; Collect. Rap. dedic. H. B. Mann. A.E. Ross, O. Taussky-Todd, 1977. P. 241-262.

26. Seitz G. M. Flag-transitive subgroups of Chevalley groups // Ann. of Math. 1973. V. 97. P. 27-56.

27. Landazuri V., Seitz G.M. On the minimal degrees of projective representations of the finite Chevalley groups // J. Algebra. 1974. V. 32. P. 418-443.

28. Seitz G. M., Zalesskii A. E. On the minimal degrees of projective representations of the finite Chevalley groups. II // J. Algebra. 1993. V. 158. P. 233-243.

29. Tiep P.H., Zalesskii A. E. Minimal characters of the finite classical groups // Comm. Algebra. 1996. V. 24. P. 2093-2167.

Институт теоретической и прикладной математики, Алматы

Поступила в редакцию 